\title{
Business intelligence and big data analytics for organizational performance management in public sector: the conceptual framework
}

\begin{abstract}
The implementation of business intelligence (BI) and big data analytic (BDA) in managing organizational performance especially in public sector is important and critical. Weakness in managing the implementation strategy and the performance can result a massive impact to people and nation. Therefore, integration of BI and BDA are necessity to assist decision makers to increase efficiency in public services. However, preliminary study had identified limited implementation of $\mathrm{BI}$ and business analytics with organisation performance management (OPM) that led to inefficient performance in management practice. At the same time, large amount of data from various resources have headed to the emergent of big data analytics. Therefore, this research is proposed with the aim to develop an integrated framework of business intelligence and big data analytics (BI-BDA) for OPM. To achieve this goal, elements and sub elements of integrated BI-BDA and OPM implementation will be identified which focuses on big data analytics. Main outcome from this research is the new integrated framework of BI and BDA (BI-BDA) for OPM in public sector. The proposed framework is valuable for the practitioners as well as the stakeholders to ensure the OPM system to be more effective and dynamic.
\end{abstract}

Keyword: Big data analytics; Business intelligence; Organizational performance management; Public sector 